\title{
Generalized Multishearing Interferometry for the Complete Multidimensional Characterization of Optical Beams and Ultrashort Pulses
}

\author{
Adam S. Wyatt ${ }^{1 *}$, Jens Biegert ${ }^{2}$ and Ian A. Walmsley ${ }^{1}$ \\ ${ }^{1}$ Clarendon Laboratory, University of Oxford, Parks Road, Oxford, OX1 3PU, UK \\ ${ }^{2}$ ICFO - Insitut de Ciencies Fotoniques, Mediterranean Technology Park, \\ 08860 Castelldefels, Barcelona, Spain \\ *a.wyatt1@physics.ox.ac.uk
}

\begin{abstract}
We demonstrate increased accuracy and precision in the reconstruction of the multidimensional phase of electromagnetic fields based on multiple spectral shearing interferometry measurements made with shears of an arbitrary magnitude.

(C) 2011 Optical Society of America

OCIS codes: (320.0320) Ultrafast optics, (320.7100) Ultrafast measurements
\end{abstract}

\section{Introduction}

The measurement of arbitrary complex electromagnetic waveforms is key to understanding and optimizing many physical processes. Shearing interferometry (SI) is a self-referencing method for measuring the gradient of the spatial/spectral phase of electromagnetic field by the interference of two replica fields that have been translated with respect to each other in the direction of the gradient. SI can be performed in the spatial domain (lateral shearing interferometry) [1], spectral domain (spectral shearing interferometry) [2] or both [3].

SI is a powerful technique since it encodes the phase gradient directly in the interferogram as a modulation in the fringe spacing resulting from the interference of the two beams, leading to a robust algebraic (non-iterative) and rapid inversion. Since the phase gradient is encoded locally, the accuracy of the phase at a given co-ordinate is proportional to intensity at that location at that location. Due to integration of the measured interferometric phase, large errors can accumulate if a small shear with respect to pupil size / pulse bandwidth is used. A large shear can be used to reduce the accumulated error, but at the expense of a reduced resolution, or equivalently increased smoothing, of the reconstructed phase. If the shear is smaller than the width of any nulls in the intensity, then the relative phase across the null is lost, limiting the smallest shear that can be used.

Austin et al [4] demonstrated enhanced precision and accuracy of the spectral phase reconstructed from multiple spectral shearing measurements whilst maintaining spectral resolution and recovering the relative spectral phase across spectral nulls. A limitation to this method is that different spectral shears are required to be an integer multiple of the smallest shear. This is not necessarily optimal or practical.

Here we generalize the multishearing method to utilize shears of an arbitrary magnitude and in multiple dimensions to provide complete reconstruction of arbitrary waveforms in multiple dimensions. Since there is no direct restriction on the size of the shears, it is not necessary to ensure an accurate and precise calibration of the shear axis of the device, or to determine the shear magnitudes a priori to the measurement. There is also no restriction on the reconstruction sampling grid; we demonstrate how the optimal sampling grid can be determined from the experimental data. We then demonstrate numerically and experimentally that the method enhances both the precision and accuracy of the reconstruction of the spectral phase of a complex pulse: the output of a hollow-core fibre pulse compressor. The accuracy of the measurements are verified experimentally by a comparison of different shear measurements, the experimental results are presented in [5].

\section{Reconstruction algorithm}

Let the measured interferometric phase, $\theta(\mathbf{x})$ where $\mathbf{x}=[x, y, \omega]$ for an arbitrary electromagnetic field, be written in terms of the unknown test phase $\boldsymbol{\theta}(\mathbf{x})$ and shear $\mathbf{s}=[\delta x, \delta y, \delta \omega]$ as

$$
\theta(\mathbf{x}, \mathbf{s})=\phi(\mathbf{x}-\mathbf{s})-\phi(\mathbf{x}) .
$$


Often it is assumed that the measured interferometric phase is approximately equal to the gradient of the test phase, scaled by the shear, i.e. $\theta(\mathbf{x}) \simeq|\mathbf{s}| \partial \phi / \partial \hat{\mathbf{s}}$. Although this provides for an intuitive understanding, this assumption is not made here. From eq. 1, it is clear that the problem can be written in terms of a set of linear equations

$$
\mathbf{W A p}=\mathbf{W t}
$$

where $\mathbf{p}=\phi(x, y, \omega)$ and $\mathbf{t}=\boldsymbol{\theta}(x, y, \omega)$ are test phase and measured interferometric phase respectively, mapped into column vectors, $\mathbf{A}$ is a shearing matrix that performs the shear operation and $\mathbf{W}$ is a diagonal weighting matrix whose elements are equal to the measured interferometric intensity. In the case of a single shear along a single dimension, matrix $\mathbf{A}$ is square bi-diagonal such that inversion of eq. 2 gives rise to the standard concatenation formula often employed in SPIDER. The phase reconstruction from multiple shears proceeds by vertically concatenating the measured interferometric phases and vertically concatenating the corresponding shear matrices, see fig. 1 (a).
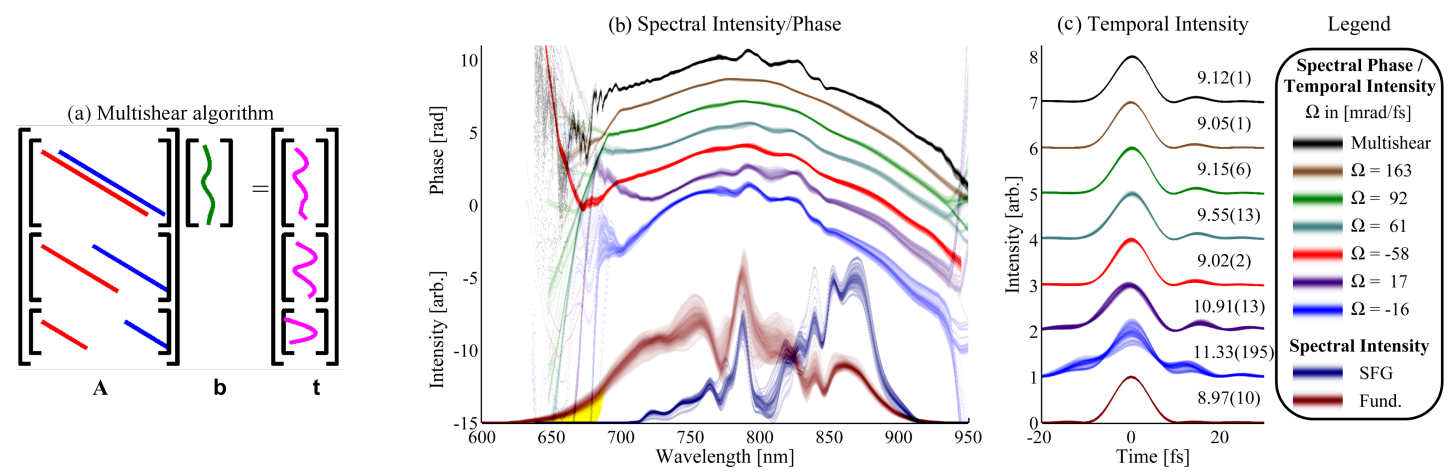

Fig. 1. (a) Pictorial representation of multishear algorithm. (b) Spectral phase reconstruction of the output of a hollow fibre for different shears. As the magnitude of the shear increases, the precision increases and accuracy decreases. (c) Temporal reconstructions from (b). Small shears are inaccurate due to the loss in the relative phase across spectral nulls.

We present two specific methods for inverting the data along multiple dimensions with multiple shears based on eq. 2: (1) modal decomposition of the unknown phase and (2) reconstruction of the unknown phase on an optimal sampling grid. A comparison of the different single shear measurements enables the accuracy of the reconstruction to be experimentally estimated, see fig. 1 (b-c).

\section{Conclusion}

In summary, we have demonstrated how to optimize the extraction of phase information from multidimensional multiple shearing interferograms using a simple least-squares algorithm. We have demonstrated via numerical and experimental analysis that the precision and accuracy of the reconstructed phase is enhanced compared to single-shear methods, without placing restrictions on the magnitude of the shears.

\section{References}

1. D. Malacara, Optical Shop Testing," (Wiley-Interscience, 2007)

2. C. Iaconis and I. A. Walmsley, "Spectral phase interferometry for direct electric-field reconstruction of ultrashort optical pulses," Opt. Lett. 23, 792-794 (1998).

3. C. Dorrer, E. M. Kosik, and I. A. Walmsley, "Spatio-temporal characterization of the electric field of ultrashort optical pulses using two-dimensional shearing interferometry," Applied Physics B - Lasers And Optics 74, S209-S217 (2002).

4. D. R. Austin, T. Witting, and I. A. Walmsley, "High precision self-referenced phase retrieval of complex pulses with multiple-shearing spectral interferometry," J. Opt. Soc. Am. B 26, 1818-1830 (2009).

5. Adam S. Wyatt, Alexander Grün, Philip K. Bates, Olivier Chalus, Jens Biegert, and Ian A. Walmsley, "Accuracy measurements and improvement for complete characterization of optical pulses from nonlinear processes via multiple spectral-shearing interferometry," Opt. Express 19, 25355-25366 (2011). 